\title{
Endoscopic retrograde cholangiopancreatography in infants and children
}

\section{(ㄷ)(1) $\odot$}

\author{
Authors \\ Tone Lise Åvitsland ${ }^{1}$, Lars Aabakken ${ }^{1,2}$ \\ Institutions \\ 1 Section for Gastroenterology, Department of \\ Transplantation Medicine, Oslo University Hospital, \\ Oslo, Norway \\ 2 Institute of Clinical Medicine, Faculty of Medicine, \\ University of Oslo, Oslo, Norway
}

submitted 11.5.2020

accepted after revision 19.11.2020

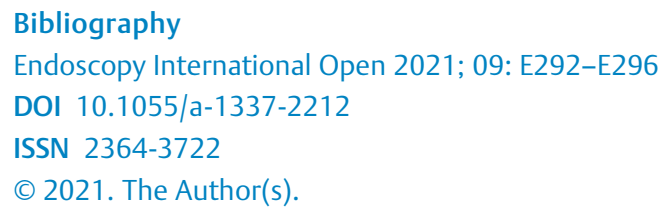
Commons Attribution-NonDerivative-NonCommercial License, permitting copying and reproduction so long as the original work is given appropriate credit. Contents may not be used for commecial purposes, or adapted, remixed, transformed or built upon. (https://creativecommons.org/licenses/by-nc-nd/4.0/)

Georg Thieme Verlag KG, Rüdigerstraße 14,

70469 Stuttgart, Germany

Corresponding author

Tone Lise Åvitsland, Section of Gastroenterology, Department of Transplantation Medicine, PO box 4950 Nydalen, 0424

Oslo, Norway

Fax: +4723070670

tavitsla@ous-hf.no

\begin{abstract}
Background and study aims Previous reports have suggested that endoscopic retrograde cholangiopancreatography $(E R C P)$ in pediatric patients are safe. However, the total number of cases presented in the literature remains small. We present results regarding safety and outcomes in pediatric patients undergoing ERCP at Oslo University Hospital.

Patients and methods Patients $<18$ years who underwent ERCP between April 1999 and November 2017 were identified using procedure codes. Medical records were examined for age, gender, diagnosis, indications, type of sedation, findings, interventions, and complications.

Results A total of 244 procedures were performed in 158 patients. Fifty-six of these were in 53 infants (age $\leq 1$ year). Mean age was 8.8 years. The youngest patient was 8 days old. Mean weight was $5.0 \mathrm{~kg}$ in infants, the smallest weighing $2.9 \mathrm{~kg}$. Cannulation failed in 19 (7.8\%). The main indication in infants was suspicion of biliary atresia $(n=38)$. Six of the procedures $(10.7 \%)$ were therapeutic. In children the main indications were biliary stricture $(n=64)$ and investigation of primary sclerosing cholangitis (PSC) $(n=45) .119$ $(63.2 \%)$ of these procedures were therapeutic.

Complications were uncommon in infants; only two episodes of infection were registered. In children ( $>1$ year) post-ERCP pancreatitis were seen in $10.4 \%$.

Conclusions Our retrospective series of ERCP procedures includes 56 procedures in infants, which is one of the largest series presented. Complications in infants are rare and post-ERCP pancreatitis was not seen. In older children $10.4 \%$ experienced post-ERCP pancreatitis. In expert hands, ERCP was shown to be acceptably feasible and safe in infants and children.
\end{abstract}

\section{Introduction}

Endoscopic retrograde cholangiopancreatography (ERCP) is an endoscopic technique used to examine and perform interventions in the pancreaticobiliary system. ERCP is routinely being used in adults, and over the last decades its use has been increasingly therapeutic, mainly due to the non-invasive imaging of magnetic resonance cholangiopancreatography (MRCP). In children however, the use of ERCP has been less frequent, due to less clinical indications, as well as specific technical challenges and limitations. Publications on ERCP in pediatric populations are still limited, but they suggest that ERCP in infants and children is both efficient and safe [1-3].

The aim of this retrospective study was to review our experience with ERCP in this group: indications, outcomes, and safety in infants and children. 


\section{Patients and methods}

All patients younger than age 18 years who underwent ERCP between April 1999 and November 2017 were identified from a prospectively kept endoscopy database. Clinical data were obtained from medical records. Age, gender, diagnosis, indications, type of sedation, findings, interventions, and complications were registered. Children $<1$ year of age were categorized as infants.

All procedures were performed by an endoscopist trained in adult ERCP. In children younger than 2.5 to 3 years, a pediatric duodenoscope with an outer diameter of $7.5 \mathrm{~mm}$ and working channel of $2.0 \mathrm{~mm}$ was used (PJF-160; Olympus Medical systems Co., Tokyo, Japan). A 5 Fr sphincterotome, Minitome (Cook Medical Inc, Bloomington IN, US) was used with the pediatric duodenoscope. In older children our standard duodenoscope with an outer diameter of $11.5 \mathrm{~mm}$ was used (TJF-160R; Olympus Medical systems Co., Tokyo, Japan) (\$Fig.1). In some of the children with a Roux-en-Y anatomy balloon enteroscopes were used. Autotome (Boston Scientific) sphincterotomes was used to perform sphincterotomy with the standard duodenoscope.

Most of the procedures were done under general anesthesia. However, in some of the teenagers, conscious sedation with midazolam/fentanyl was used.

Failed cannulation or other failure to complete the planned procedure were defined as a failure.

Post-ERCP pancreatitis (PEP) was defined as the occurrence of typical pain and elevation of amylase in serum more than three times normal levels. Severity was defined and graded according to the consensus criteria developed by Cotton et al [4].

Results are presented as mean, standard deviation (SD), range and percentages as appropriate.

The study was categorized as a quality assurance project, and thus did not require Ethics Committee approval. It was, however, authorized by the Internal Research Review Board.

\section{Results}

A total of 244 procedures were performed in 158 children. Patient age ranged from 8 days to 17.9 years (mean 8.8 years, SD 6.43 ). Patients' weight ranged from $2.9 \mathrm{~kg}$ to $83.0 \mathrm{~kg}$ (mean $32.8 \mathrm{~kg}$, SD 24.9) (missing data from 38 procedures). 56 procedures were performed in 53 infants $(\leq 1$ year, mean age 3 months, SD 0.22). Mean weight in infants was $5.0 \mathrm{~kg}$ (range $2.9 \mathrm{~kg}$ to $9.0 \mathrm{~kg}$, SD 1.4) (missing data from 6 procedures). Two infants underwent 2 and 3 procedures, respectively. Both underwent ERCP due to biliary leakage, one after liver transplantation and one spontaneous. 188 procedures were performed in 105 children between ages 1 to 18 years. - Table 1 shows the age distribution. Several patients underwent more than one procedure ( $\triangleright$ Fig. 2 ). In two procedures in infants and 38 procedures in children the patient had a Roux-en-Y anatomy with hepaticojejunostomy.

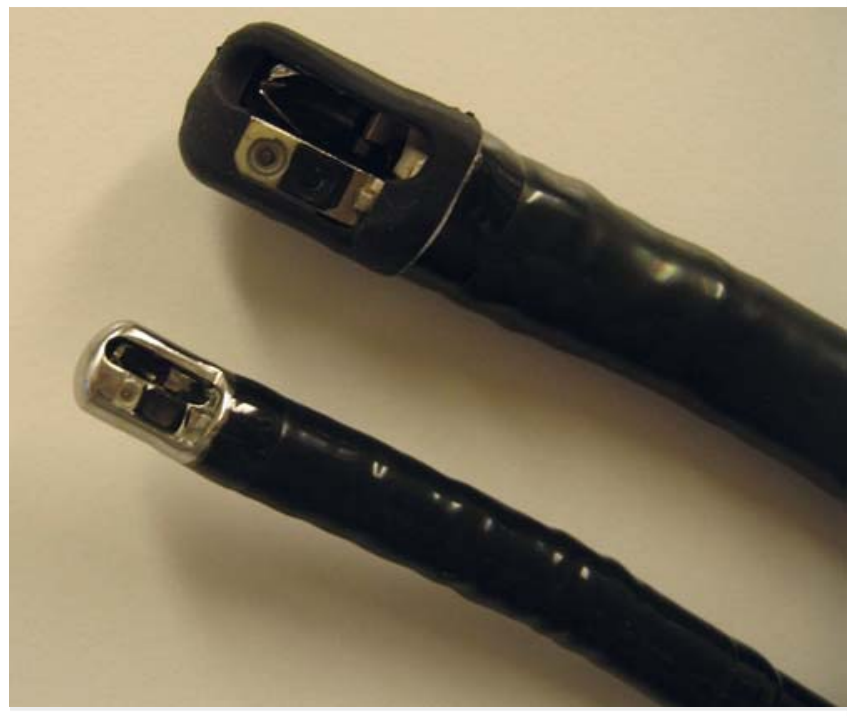

- Fig. 1 A pediatric duodenoscope (PJF160) alongside a standard TJF160 R Olympus duodenocope (top).

- Table 1 Number of procedures in different age groups.

\begin{tabular}{|l|c|}
\hline Age (years) & Procedures, $\mathbf{n}(\%)$ \\
\hline$\leq 1$ & $56(23)$ \\
\hline$>1$ to $\leq 2$ & $10(4)$ \\
\hline$>2$ to $\leq 5$ & $23(9)$ \\
\hline$>5$ to $\leq 12$ & $53(22)$ \\
\hline$>12$ to $<18$ & $102(42)$ \\
\hline Total & 244 \\
\hline
\end{tabular}

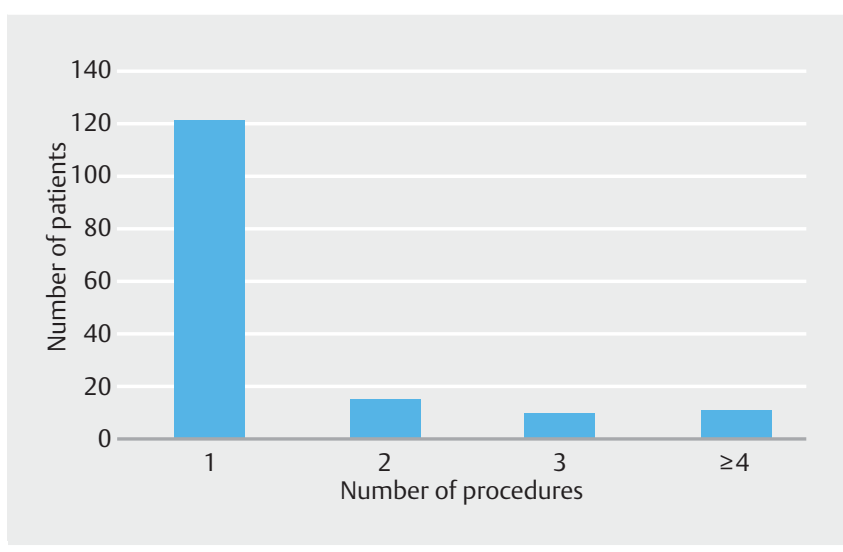

- Fig. 2 Number of procedures per patient.

\section{Indications}

The predominant indication in infants $(38 / 56)$ was cholestasis with suspicion of biliary atresia ( $\downarrow$ Table 2 ). We performed ERCP in infants with suspected biliary atresia where non-invasive diagnostic work-up such as ultrasound and magnetic resonance cholangiography (MRCP) were inconclusive. In 21 of the 


\begin{tabular}{|l|c|c|}
\hline \begin{tabular}{l} 
Table2 ERCP indications. \\
\hline Indication
\end{tabular} & $\begin{array}{l}\text { Infants, } \mathbf{n} \\
(\%)\end{array}$ & $\begin{array}{l}\text { Children, } \mathbf{n} \\
\text { (\%) }\end{array}$ \\
\hline Biliary atresia & $38(67.9)$ & - \\
\hline Biliary stricture & $2(3.6)$ & $64(34.0)$ \\
\hline Primary sclerosing cholangitis & - & $45(23.9)$ \\
\hline Biliary stone & $4(7.1)$ & $24(12.8)$ \\
\hline Biliary leakage postoperative & $5(8.9)$ & $9(4.8)$ \\
\hline Choledochal cyst & $5(8.9)$ & $8(4.3)$ \\
\hline Chronic pancreatitis & & $11(5.9)$ \\
\hline Acute pancreatitis & $1(1.8)$ & $3(1.6)$ \\
\hline Hyperbilirubinemia & $1(1.8)$ & $8(4.3)$ \\
\hline Pancreatic duct stricture & - & $5(2.7)$ \\
\hline Traumatic liver/pancreatic injury & - & $7(3.7)$ \\
\hline Pancreatic/biliary tumor & - & $2(1.1)$ \\
\hline Papillary dysfunction & - & $1(0.5)$ \\
\hline Cryptosporidiosis & - & $1(0.5)$ \\
\hline Total & 56 & 188 \\
\hline
\end{tabular}

38, ERCP findings suggested biliary atresia. Of these, 17 underwent surgery with portoenterostomy and two underwent liver transplantation. Two infants in whom ERCP suggested biliary atresia turned out to be false-positive; one underwent exploratory laparotomy and biliary atresia was excluded, and one was diagnosed with $\alpha$-1-antitrypsin deficiency before surgery was performed. More importantly, ERCP findings excluded biliary atresia in 16 of 38 infants, obviating the need for exploratory surgery. In one infant the papilla looked normal, but cannulation failed. The endoscopist concluded that biliary atresia was unlikely. However, further examination with ultrasound supported the diagnosis of biliary atresia and exploratory surgery confirming biliary atresia and subsequent portoenterostomy was performed. In our cohort, we found a sensitivity of $95 \%$ and a specificity of $89 \%$ of ERCP for biliary atresia.

In children there were several equally common indications, mostly involving the biliary system and less commonly the pancreatic system ( $\triangleright$ Table 2 ). In 64 cases the indication for ERCP was either suspected or known biliary stricture. Only five strictures were categorized as benign with different etiology, one patient with two procedures had distal choledochal stricture of unknown etiology, one had suspected IgG4 cholangitis and no stricture was found, one had autoimmune pancreatitis with stent placement due to stricture, and one had cirrhosis with minor dilation of choledochus and underwent sphincterotomy. The remaining 59 were postoperative strictures, mainly in patients who had undergone liver transplantation; however, one child with progressive familial intrahepatic cholestasis had a draining interponate between the gallbladder and colon, suspected to be occluded.
In patients with PSC, the main indication for ERCP was to confirm or exclude PSC. Many of these patients had undergone an MRCP that was suspicious for or inconclusive concerning PSC. In only 15 cases the indication was increasing symptoms or MRCP findings of dominant strictures. In these cases, ERCP was performed for therapeutic intent. In total, brush cytology was performed in five cases.

In 11 children the indication for ERCP was chronic pancreatitis. The procedure was performed to exclude microlithiasis, exclude pathology of the papilla, pancreatic stent insertion, pancreatic stone removal, and treat incomplete pancreatic divisum. The indication for ERCP was acute pancreatitis in three children, and the intention was to exclude pancreatic duct stenosis, papillary stenosis/dysfunction and an anatomical substrate, such as pancreatic divisum. In one infant ERCP was performed due to acute pancreatitis to rule out papillary stenosis and an anatomical substrate. Cannulation failed; however, a precut was performed.

In eight children and five infants, the indication for ERCP was choledochal cyst. In these patients, the procedure was performed to clarify the anatomy of the biliary tract, e. g. identify a common channel or other insertions of the pancreatic duct, to optimize surgery.

\section{Failed procedures}

The success rate in our study was $92.2 \%$. In infants, cannulation failed in six $(10.7 \%)$ cases. Two of these children had Roux-en-Y anatomy after liver transplantation and we failed to reach the hepaticojejunostomy. In two infants aged 8.4 and 1.2 months, respectively, cannulation failed due to the combination of a large instrument and small duodenum. In one we failed to cannulate despite reasonable anatomical access. In the last one, the intrahepatic bile ducts were not visualized, but leakage from the cystic duct was shown, which made this examination partially successful.

In children, biliary access failed in 13 (6.9\%). In eight of these patients, we were unable to reach their hepaticojejunostomy after previous Roux-en-Y reconstruction. In two, we failed to cannulate the native papilla, and in three the examination had to be discontinued due to sedation concerns. In five more cases the pre-procedural intentions were only partially resolved.

\section{Endoscopic therapy}

In infants, interventions were performed in six procedures $(10.7 \%)$; sphincterotomy in five and stent removal in one. In one, sphincterotomy with subsequent stone extraction and biliary stenting was done. In children, 119 of the procedures were therapeutic (63.2\%), and 176 interventions were performed $(\triangleright$ Table 3$)$. Sphincterotomy was performed in 39 cases.

\section{Complications}

A total of 24 complications were seen in 222 procedures (10.8\%) (missing data from 22 procedures) ( $\checkmark$ Table 4$)$ ). Complications were infrequent in infants. There were no cases of PEP. One infant had a small elevation of serum amylase and due to fever and $\mathrm{C}$-reactive protein level reaching 60 , antibiotics was given 
- Table 3 ERCP interventions.

\begin{tabular}{|l|l|c|}
\hline Intervention & Infants, $\mathbf{n}$ & Children, \\
\hline $\begin{array}{l}\text { Sphincterotomy (biliary, pancreat- } \\
\text { ic, anastomotic) }\end{array}$ & 5 & 39 \\
\hline Stricture dilation & - & 41 \\
\hline Biliary stone extraction & 1 & 17 \\
\hline Stent placement & 1 & 43 \\
\hline Stent removal & 2 & 23 \\
\hline Placement of naso-biliary tube & - & 7 \\
\hline Brush cytology & - & 5 \\
\hline Pancreatic stone extraction & - & 1 \\
\hline Total number of interventions & 9 & 176 \\
\hline Total procedures with interventions & $6 / 56(10.7 \%)$ & $119 / 188$ \\
\hline
\end{tabular}

two days after ERCP. Another infant developed fever shortly after ERCP and antibiotics were given. Planned surgery was postponed for 10 days.

Of the children, $10.4 \%$ (18/173) experienced PEP. Of the 39 patients in whom a sphincterotomy was performed, six (15.4\%) experienced PEP, two of them graded as moderate and four mild. After stricture dilation four children had PEP, three mild and one moderate. One of these patients also experienced bleeding, which was managed conservatively. Three children had mild PEP after placement of naso-biliary tube. Five children had PEP after diagnostic ERCP, one of which was moderate. The indication for ERCP was PSC in three of these cases.

\section{Discussion}

ERCP has different utility in different age groups. In infants it is still mainly a diagnostic procedure, and the main role in our series was to confirm or exclude biliary atresia. Although ERCP is not routinely recommended as part of the work-up of neonatal cholestasis, it is beneficial in selected patients and may obviate explorative laparotomy [5-7]. In infants with suspected biliary atresia we found that ERCP had a sensitivity of $95 \%$ and a specificity of $89 \%$. In a previous study of ERCP procedures in cholestatic infants and neonates the sensitivity of ERCP in the diagnosis of biliary atresia was shown to be $86 \%$, with a specificity of $94 \%$ [8]. The sensitivity and specificity of biliary atresia diagnosis in another series were $92 \%$ and $73 \%$, respectively [9]. We had a false-positive result in two of 21 (9.5\%) infants. In comparison, Keil et al had a false-positive result in 2 of 38 (5.3\%) cases [8].

Failed cannulation was seen in $10.7 \%(6 / 56)$ infants. This number compares well to other materials $[1,7,8,10]$. In one patient the endoscopist described a normal papilla but failed to cannulate. The remaining examinations suggested biliary atresia, which was subsequently confirmed. Keil et al found that of nine failed cannulations eight were later diagnosed with biliary atresia [8]. There were no severe complications
- Table 4 Complications after ERCP.

\begin{tabular}{|l|c|c|}
\hline Complications & Infants, $\mathbf{n}$ & Children, $\mathbf{n}$ \\
\hline Pancreatitis (any) & - & 18 \\
\hline Mild & - & 14 \\
\hline Moderate & - & 4 \\
\hline Severe & - & - \\
\hline Infection/cholangitis & 2 & 2 \\
\hline Bleeding & - & $1^{1}$ \\
\hline $\begin{array}{l}\text { Perforation without clinical } \\
\text { symptoms }\end{array}$ & - & 1 \\
\hline Total & $2 / 49(4.1 \%)$ & $22 / 173(12.7 \%)$ \\
\hline 1 This patient also experienced a mild pancreatitis. \\
\hline
\end{tabular}

after ERCP in infants. Interestingly, we did not see PEP in infants. Shanmugam et al also reported no pancreatitis in infants younger than 100 days [7]. To our knowledge there are no good hypotheses to explain why PEP is seldom seen in infants. However, one should expect that since the use of guidewire is not possible in infants, because the Minitome is too small for both contrast and a wire, the cannulations would be more difficult, thus increasing the risk of PEP.

In children between 1 and 18 years, $63.2 \%$ of ERCP procedures were therapeutic, which is comparable to other publications $[1,11,12]$. PEP was seen in $10.4 \%$, which is similar to others $[1,12,13]$. However, other studies have demonstrated lower rates of pancreatitis [10]. There were no cases of severe pancreatitis and no mortality after ERCP. In adults both prophylactic pancreatic duct stenting and rectal nonsteroidal anti-inflammatory drugs have been shown to prevent PEP. In children, the effect of these measures is not well studied [14]. The infants and children in this study did not receive nonsteroidal anti-inflammatory drugs or prophylactic pancreatic duct stenting. One could argue that $10.4 \%$ cases of PEP are a high number, however, most of them were mild, and none were severe. We think that this number is acceptable when alternative treatment may be surgery with possible even higher risk for complications. Five cases of PEP in our population occurred after diagnostic procedures, which are less acceptable. That was especially true in three of the cases for which the intention was to confirm or rule out PSC. In retrospect, ERCP should have been avoided in these patients, since MRCP is the diagnostic modality of choice in these patients. In general, the indication for ERCP in patients with PSC should mainly be to treat strictures or perform brush cytology. Due to the overall small number of PEP, we did not find any risk factors. However, difficult cannulation and unintended pancreatic passage could be factors in some of the cases, and interventions, such as sphincterotomy, dilation and placement of naso-biliary drainage, are other possible risk factors in our population. In general, both patient and technical factors may increase the risk of PEP. It should be emphasized that ERCP in children is difficult and should only be performed by experienced endoscopists. Most of the proce- 
dures were performed by two expert endoscopists in our center, which possibly is one of the reasons for the low number of complications and failures seen, especially in the infants. In small children, one needs to especially consider the possibility of compression of the large vessels during endoscope insertion. Other technical difficulties are limited navigational space and respiratory restriction due to small bowel gas distension during the procedure, even with the use of $\mathrm{CO}_{2}$. This limits the time available for the procedure. The normal-sized duodenoscope has limitations, and in the smallest children ( $<2-2.5$ years) a pediatric duodenoscope is needed. The American Society for Gastrointestinal Endoscopy Technology Committee recommend adult duodenoscopes in patients $>10 \mathrm{~kg}$ [14]. In our material we used a pediatric duodenoscope in infants. However, the $2.0-\mathrm{mm}$ instrument channel limits the available accessories, making some interventions difficult to perform. At the moment, the pediatric duodenoscope is not being produced, and future development is uncertain.

\section{Conclusion}

To conclude, ERCP can be helpful in diagnostic work-up of cholestasis in infants. In older children, ERCP is mainly therapeutic, similar to adults. Complications in infants are rare. In children, complications such as pancreatitis are more common. However, in expert hands, ERCP was shown to be acceptably feasible and safe in infants and children.

\section{Competing interests}

The authors declare that they have no conflict of interest.

\section{References}

[1] Keil R, Drabek J, Lochmannova J et al. ERCP in infants, children, and adolescents-Different roles of the methods in different age groups. PLoS One 2019; 14: e0210805

[2] Rosen JD, Lane RS, Martinez JM et al. Success and safety of endoscopic retrograde cholangiopancreatography in children. J Pediatr Surg 2017; 52: 1148-1151

[3] Kohoutova D, Tringali A, Papparella G et al. Endoscopic treatment of chronic pancreatitis in pediatric population: Long-term efficacy and safety. United European Gastroenterol J 2019; 7: 270-277

[4] Cotton PB, Lehman G, Vennes J et al. Endoscopic sphincterotomy complications and their management: an attempt at consensus. Gastrointest Endosc 1991; 37: 383-393

[5] Aabakken L, Aagenaes I, Sanengen T et al. Utility of ERCP in neonatal and infant cholestasis. J Laparoendosc Adv Surg Tech A 2009; 19 : 431-436

[6] Troendle DM, Barth BA. Pediatric considerations in endoscopic retrograde cholangiopancreatography. Gastrointest Endosc Clin N Am 2016; 26: 119-136

[7] Shanmugam NP, Harrison PM, Devlin J et al. Selective use of endoscopic retrograde cholangiopancreatography in the diagnosis of biliary atresia in infants younger than 100 days. J Pediatr Gastroenterol Nutr 2009; 49: 435-441

[8] Keil R, Snajdauf J, Rygl M et al. Diagnostic efficacy of ERCP in cholestatic infants and neonates - a retrospective study on a large series. Endoscopy 2010; 42: 121-126

[9] Petersen C, Meier PN, Schneider A et al. Endoscopic retrograde cholangiopancreaticography prior to explorative laparotomy avoids unnecessary surgery in patients suspected for biliary atresia. J Hepatol 2009; 51: 1055-1060

[10] Giefer M], Kozarek RA. Technical outcomes and complications of pediatric ERCP. Surg Endosc 2015; 29: 3543-3550

[11] Usatin D, Fernandes M, Allen IE et al. Complications of endoscopic retrograde cholangiopancreatography in pediatric patients; a systematic literature review and meta-analysis. J Pediatr 2016; 179: 1605 e3

[12] Felux J, Sturm E, Busch A et al. ERCP in infants, children and adolescents is feasible and safe: results from a tertiary care center. United European Gastroenterol J 2017; 5: 1024-1029

[13] Kieling CO, Hallal C, Spessato CO et al. Changing pattern of indications of endoscopic retrograde cholangiopancreatography in children and adolescents: a twelve-year experience. World J Pediatr 2015; 11: 154-159

[14] Committee AT, Barth BA, Banerjee $S$ et al. Equipment for pediatric endoscopy. Gastrointest Endosc 2012; 76: 8-17 